\title{
The Elderly-oriented Design Exploration of Zhangzhou Residential Community
}

\author{
Bo Li \\ Xiamen University Tan Kah Kee College; Zhangzhou 363105 China
}

Keywords: Design suitable for the elderly; Residential community; Ageing; Zhangzhou.

\begin{abstract}
To the problem of aging population as the background, from the perspective of community residence suitable for the old design, analysis of regional characteristics, space types, functional requirements, and reference the excellent examples of similar city residential communities as the philosophy basis and reference, and then discuss under the background of the aging community fit the old residential design, try to provide effective ways for its design..
\end{abstract}

\section{Introduction}

At present, the development of domestic elderly care sector is in the ascendant and social concern that continues to heat up. Aging population is increasing, especially in the elderly over eighty years old, increasing at the rate of million, therefore life problems of the elderly must be taken seriously. The domestic situation of traditional family pension model cannot fully meet the needs of modern families, and the development of social pension institutes has their own difficulties. The more obvious is a serious lack of design for aging, so the pension space is less satisfied. Elderly community care is closely related with residential interior and exterior design. Thus, a scientific space design, a suitable environment for the aging, a good use of space aesthetics and functional requirements are the starting points of this research. At present, the study on space of community housing in domestic scholars is not systematic from the perspective of the elderly, which leads that the design is not in depth at design hierarchy and other issues. The study on space of community housing is relatively simple, staying in the general principles, and there are many research blanks on community housing, including study on suitable environment for the aging, and targeted research on the characteristics of different construction period of community housing. In this paper, the author studied and draw on the mature form of community housing, choosing the model suitable for Zhangzhou development and trying to fill the blank of the study of community housing in Zhangzhou before, to have a systematic classification and research on community residential space at multi-level and multi-angle, so that having a targeted research on the design for aging of community housing in different ears.

\section{Current Situation and Problems of Community Housing in Zhangzhou}

\subsection{Current Situation}

The population aging in Zhangzhou, as a region with higher life expectancy, shows the characteristics of rapid in growth and high in aging level. The younger elderly population is in the majority of the population, but the trend of population aging is obvious. In the 1980s and 1990s, the construction of the community and its public facilities were relatively scarce, and the space resources were insufficient. Zhangzhou built community pension scheme from 2009, starting relatively late, and the development was relatively slow. As the economic development is relative slow, the investment on the community pension funds is inadequate for a long time, only by the community to build their own, and the community cannot afford the normal operation of pension space such as nursing homes, the development of community pension construction is slow. According to survey, the general concept of the elderly in the region is relatively conservative, they feel that they should be at home, and do not want to live in community housing (nursing home, etc.), while the existing community pension space cannot meet the actual needs of the elderly, so the majority of the pension space in community is not divided. Therefore, the different activity space in community housing should be placed together, making it have a composite function. 


\subsection{Elderly-Oriented Design Problems}

At the beginning of construction, the majority of communities does not take into account any elderly-oriented design or relative application requirement. In response to requests from elderly people, unused space in a minority of communities has been changed into special recreation rooms for the elderly. However, recreation rooms are not sufficient to satisfy their demand. At present, most of the residential communities in Zhangzhou, including those built in the early 1980s, are not equipped with any elderly-oriented design product or whose facilities for the elders cannot meet the existing huge demand. It is urgent to have a plan for elderly-oriented design and to commence the construction. Therefore, a large number of residential buildings will not be eliminated based on the condition that our country is completely entering an aging society in ten years. People with elderly family members can still live there and a waste of economy and resources would be prevented.

\section{Introduction and Analysis on Categories of Elderly-Oriented Space Design}

\subsection{Space Units in Residential Communities}

The main categories of space units in residential communities should include the bedroom and the restroom, so basic living needs of the elderly could be guaranteed. As for someone with a self-care ability, the kitchen and the living room are equipped in addition to maintain their normal daily activities.

\subsection{Public Space in Residential Communities}

Public space in residential communities has a variety of categories, which should include the canteen, the reading room, the chess room, the multi-function room, the fitness room, the clinic, the grocery store, the mail room, the barber shop, the laundry, the administration office, the storeroom, the front office and the corridor etc. If space permitting, we can have religious activities space, such as the prayer room, to promote our Chinese traditional culture.

\subsection{Analysis on Functional Organization of Residential Communities}

We are not merely aiming at creating a warm environment for old people by applying elderlyoriented design , but also making efforts in cultivating their diverse interests and hobbies. Therefore, people 's spiritual life could be renewed and enriched and they are going to live a better life with refreshment of mind and body. The design of space unit should focus on stabilization and normalization, making people feel coziness and comfortable. Safety systems and medical equipment can be installed within the bedroom for the convenience of old people. At the same time, interest and aesthetics factors can not be ignored during the process of space design. Considering physical and psychological characteristic of old people, here are some suggestions for public space design: First, the classroom can be divided from cultural learning space and combined with other space like the reading room, the painting and calligraphy room, the hall or other multi-purpose space. The second, the sports fitness space can be built as an independent setting, merging as part of the rehabilitation room. Other categories of space combined can be the reading room, the multi-purpose hall and the shared living room. The third, as for the design of recreation and entertainment space, it is suggested to merge the inner circulation space, the living space and the multi-purpose hall. The lounge is usually located beside the corridor and the hallway. Moreover, the scenery window is recommended to install for the elderly. For some of them are tending to have some physical problems such as immobility as their age increases. Therefor, they need the scenery window through which they can enjoy the charming and picturesque scenery outside.

\section{The Discussion of Elderly-Oriented Design}

\subsection{The Analysis and Reference of Excellent Instances}

The project of "many generations living in the same house" of Germany is the mature space characteristic of German social service system for the aged, owing to the correspondence with the spiritual culture demand of the aged via the construction of living space environment construction. And the space environment construction of this German project is on the theory basis of society change theory, regulating the "economic man" characteristic of subjective and objective of the social 
service system for the aged, non-material attribute of service content and the mutual benefit of principle. And its space environment construction pattern is divided according to the property ownership and degree of participation, highlighting its application with the public space. Interaction between generations is the essence of the space environment construction of this German project. And the service construction of social service in our country can draw lessons from the space environment construction of German "many generations living in the same house" on the content, method and carrier.

The social service system in England has established the relatively perfect and mature system through long-term development. Although they also are faced with some problems in the aspect of social service system for the aged, they also can provide us lots of enlightenment, including: perfecting and improving the policies and regulations and standard system, devoting major efforts to introducing the folk capital, giving full expression to the market strength, enhancing the professional level of nursing institution, accelerating the talent team construction of social service for the aged, intensifying and perfecting the mechanism of supervision and management and one-step intensification of home-based care and community-based care.

\subsection{The Effective Method and Approach If Elderly-Oriented Design \\ 4.2.1The Space Size of Elderly-Oriented Design}

Along with the increasing of age, the body mechanism of the aged will gradually decline and the size of the aged is different with that of the young. Elderly-oriented design has certain particularity and it belongs to the tailor-made to do the product design. The bedroom of the aged has varieties of functions, so the area is relatively big than the ordinary bedroom to avoid the injury and striking and reserve the spare for the later life. The area of bedroom is also proper to not big, because that the too big space will express the empty feeling and intensity the coldness and loneliness, and it is relatively reasonable that the area is equal to or larger than $25 \mathrm{~m}^{2}$. Given the aged with the self-care ability, they can be allocated with one living room apartment with kitchen and bath, and it is suggested that the area is equal to or larger than $35 \mathrm{~m}^{2}$. The valid width of corridor is no less than $1.5 \mathrm{~m}$, and there must be no less than $1.5 \mathrm{~m} \times 1.5 \mathrm{~m}$ wheelchair rotation space at the both sides of the corridor. The layer height of space is not proper to be too high, and the appropriate height is about $2.7 \mathrm{~m}$. Partition should guarantee the good deafening effect and it is suggested to use the gypsum board partition (thickness is equal to or larger than $120 \mathrm{~mm}$ ) and light block partition (the thickness is around $120 \mathrm{~mm}$ ). The material selection of wall facing is not proper to choose the tough, rude and reflective materials, and the corner should shape to the round angle or be processed with soft package. And the ground choose the non-skid and easy to clear materials in order to increase the walking safety of the aged. The top surface shows with the shape of smooth and precise, adopting the lightweight and acoustic material. The height of baseboard is around $90 \mathrm{~mm}$, and its material selection lives up to the entire base line, vertical and flush with the wall space to avoid protruding the trip. Handrail is no lower than $1.1 \mathrm{~m}$, and the setting height of armrest single layer is $820 \mathrm{~mm}$, and the double-layer height is $650-900 \mathrm{~mm}$. The valid width of access door is no less than $1.5 \mathrm{~m}$, and the clear width of indoor is larger than $1 \mathrm{~m}$. Hardware chooses the U-type doorknob and pressure handling, and it is easy for the aged to seize. The most proper temperature of the aged in winter is $18-25^{\circ} \mathrm{C}$, and the humidity is $30 \%-80 \%$. Of course, lighting and ventilating are more healthy. The common vision of the aged is reducing and they have higher requirement with the illuminance, the local lighting also should be considered besides the total lighting, for example, beneath the hall and shoebox, kitchen operation platform, above the pool and open the lighting in the wardrobe. At the same time, the night-light can be set in the bedroom, and its height should have no bad effect on sleeping, and there should be keyboard light in the corner and existing attitude difference in the space to ensure some light hints and convenient for the safety of the aged getting out of the bed. Sanitary appliance should consider the entrance an turnover of wheelchair, and the height of toilet should be no lower than $0.4 \mathrm{~m}$, and the shower wheelchair of $0.45 \mathrm{~m}$ should be designed in the shower.

\subsubsection{Space Color of Elderly-Oriented Design}

The selection of space color should meet the demand of the aged, and the entire hue of bedroom is fresh and elegant, with ease and comfort, expressing the peaceful attitude. The color selection of 
furnitures should be consistent with space hue, and the armrest and handle can be selected with bright color. In order to realize the high recognition and convenience and avoid injury or fall. As shown in the material, it is easy for the aged to distinguish the yellow department and red department, and these colors can make the room seem to be bright. The color of public space prefers to the primitive, prudent and calm hue, while the consulting room prefer to the elegant hue. Medical staffs and service staffs are suggested to mainly choose the light green and pink, which can give people the quiet, warm and graceful feeling.

\subsubsection{The Identification System of Elderly-Oriented Design}

On the basis of meeting the fundamental function of identification, imagination can be fully expressed to abstract and purify the method of design element, making it interesting and full of love on the condition that the identification meets with the function. And color block element can be used to express the identification pattern, and the proper color block is beneficial for the age to distinguish. There still exists many space that they can increase, for example, normalization, systematicness and innovativeness, etc.

\section{Conclusion}

The one hand of elderly-oriented design is the requirement of the physiological changes to the housing charge, and the other hand is the requirement of the mental changes to the housing design. The research of this paper hopes to arouse the emphasis and thinking of more designers to this kind of space and provide valuable reference for the elderly-oriented design of Zhangzhou community housing.

\section{Acknowledgments}

Zhangzhou philosophy and social science project: Study on the development strategy of the Xiamen Harbour south urban morphology and creative industry parallel (NO. 2014-7-56);

Xiamen social science project: Research on the setting and optimization of Xiamen urban street furniture_ _ based on the perspective of urban experience, Xiamen City Federation of social science circles (2016) No.20

\section{References}

[1]. [Yanmin Zhou. Eged housing [M]. Beijing: China Construction Industry Press, 2011.

[2]. Jingyi Lin. The functional space allocation study of aged care facility [D]. Tsinghua University, 2011.

[3]. Molin Wanf, Jianhong Li. The first exploration of aged facility public space in Haixi district [J]. Huazhong Architecture, 2011, 29(8).

[4]. Ping Zhang, Shenmao Yang, Junmin Liu. The research of housing system construction of "supporting the aged in the housing" [J]. Modern city research. 2013.06.

[5]. Hongli Zhu, Jingyuan Li. Brief talk of the effect of mental factor on the housing interior design [J].2008. 